\title{
The role of clinical guidelines for the management of severe acute pancreatitis
}

\author{
Magdalena Gibas-Dorna' ${ }^{1}$ Jacek Piątek ${ }^{1}$, Kinga Mikrut ${ }^{1}$, Małgorzata Wojciechowska², Hanna Krauss ${ }^{1}$ \\ 'Department of Physiology, Poznan University of Medical Sciences, Poznan, Poland \\ ${ }^{2}$ Department of Mother and Child Health, Poznan University of Medical Sciences, Poznan, Poland
}

\begin{abstract}
Objective: To present the case of severe acute pancreatitis and identify some of the deviations from practice guidelines, that could negatively affect treatment-related outcomes.

Clinical presentation: A 66-year-old male presented at the city hospital with jaundice, fever and abdominal pain. Acute pancreatitis was diagnosed, but not properly classified, and open cholecystectomy with choledochotomy was performed. The patient developed multiple systemic and local complications and his condition worsened. The clinical condition was stabilized after long-term hospitalization (over 40 days) in Emergency Department and Clinic of Gastroenterology, but the recovery was not complete.

Conclusions: Patients with acute pancreatitis caused by gallstones require careful preoperative assessment according to the current established recommendations.
\end{abstract}

KEY WORDS: severe acute pancreatitis, clinical guidelines, surgical treatment.

ADDRESS FOR CORRESPONDENCE: Magdalena Gibas-Dorna, MD, PhD, Department of Physiology, Poznan University of Medical Sciences, 6 Święcickiego St., 60-781 Poznan, Poland, phone: +48 61854 65 40, e-mail: physioplus@wp.pl

\section{INTRODUCTION}

Acute pancreatitis (AP) is defined as an acute inflammatory process in the pancreas that may involve extrapancreatic inflammation including peripancreatic tissues and distant organs (particularly shock, pulmonary insufficiency, and renal failure). The clinically based classification system for AP was established in 1992 in Atlanta. Criteria for severity included organ failure and/or local complications, and were based on the Ranson signs and APACHE II scale. Based on the latest revision of this classification AP presents with three forms: mild, moderate and severe. In the severe form patients develop persistent organ failure, local complications such as peripancreatic fluid collections, pancreatic and peripancreatic necrosis, pseudocyst and walled-off necrosis [1]. Proper patient classification is crucial to appropriate and effective management.

The purpose of this paper was to present critical analysis of the acute pancreatitis misclassification and early management based on case report. We found interesting and important to describe apparent departure from the world standards and possible consequences related with it. Our intention was to indicate the importance of being familiar with current guidelines and recommendations in field, especially in the emergency situations. Finally, we wanted to provoke the discussion about world recommendations, physician experience, practice and reality.

\section{CLINICAL PRESENTATION}

A 66-year-old male patient with a past history of hypertension and cardiac infarct was admitted to the hospital with jaundice, fever and severe abdominal pain radiating to the back, unresponsive to the regular painkillers and spasmolytics, lasting 3 days. He had no history of pancreatitis or other risk factors that would have caused him to develop the underlying condition. At initial presentation his serum lipase concentration was greater than $500 \mathrm{U} / \mathrm{l}$, amylase concentration was $569 \mathrm{U} / \mathrm{l}$ in serum, and $3022 \mathrm{U} / \mathrm{l}$ in urine. Other laboratory abnormalities showed leukocytosis (16.5 G/l), anemia (Hb $9.5 \mathrm{~g} / \mathrm{dl}$, RBC $3.06 \mathrm{~T} / \mathrm{l}$, Htc 29\%), elevated serum urea (14.82 mmol/l), and serum creatinine $(465.87 \mu \mathrm{mol} / \mathrm{l})$. In addition, abdo- 
minal ultrasound, as described by surgeon, demonstrated oedematous type of pancreatitis, gallstones, and gallbladder inflammation. Based on the clinical presentation and laboratory findings, the patient was diagnosed as having acute biliary pancreatitis. The patient was treated with routine open cholecystectomy and choledochotomy. The final diagnosis was made at time of surgery: exudative-necrotizing acute pancreatitis, diffuse peritonitis, cholelithiasis and choledocholithiasis. Pharmacological management included infusion fluids, cefuroxime, ascorbic acid, and analgesics. On day 5 of his treatment, the patient's condition worsened and was described as a "psychotic behaviour" caused by uremic encephalopathy.

The patient was redirected to the emergency department (ED) in a hospital $30 \mathrm{~km}$ away for dialysis therapy.

The patient's admission laboratory findings were: serum urea $30.2 \mathrm{mmol} / \mathrm{l}$, creatinine $500.1 \mu \mathrm{mol} / \mathrm{l}, \mathrm{Hb} 6.0$ mmol/l, Htc 0.28 l/l, RBC 3.18 T/l, WBC 15.1 G/l, blood sugar $7.4 \mathrm{mmol} / \mathrm{l}$, acid-base balance: $\mathrm{pO}_{2} 38 \mathrm{~mm} \mathrm{Hg}, \mathrm{pCO}_{2}$ $47 \mathrm{~mm} \mathrm{Hg}, \mathrm{HCO}_{3}{ }^{-}$15.7, $\mathrm{BE}-7.9$, pH 7.1. Since biochemical tests did not meet an absolute indications for dialysis, the dialysis therapy was reconsidered and not initiated.

During the first few days in ED the patient's condition was described as unstable because of the following symptoms: intermittent fever up to $38.2^{\circ} \mathrm{C}$, tachyarrhythmia with atrial flutter on day 6 , increases of blood pressure up to $190 / 100 \mathrm{~mm} \mathrm{Hg}$, weakness, reduced level of consciousness, and respiratory failure with severe hypoxemia and pleural effusion. Computerized tomography $(\mathrm{CT})$ showed enlargement of the pancreas with areas of necrosis at the pancreatic head, and inflammatory peripancreatic exudation with largest fluid collection (115 cm in length) between body of the pancreas, pancreatic tail, and stomach. Additionally CT scan revealed a massive both sided pleural effusion.

Patient received total parenteral nutrition and was treated with oxygen, digoxin (after unsuccessful electrical cardioversion), metronidazole, doxycycline, fluconazole, cilastatin, insulin, omeprazole, cocarboxylasum, potassium, furosemide, metoclopramide, acetylcysteine, ascorbic acid, pentoxiphillin, and infusion fluids.

After achieving relative clinical stability, patient was transferred to the higher level of care, Clinic of Gastroenterology on day 11, where the enteral nutrition was immediately administrated. During the hospital stay patient was treated with: probiotics, pancreatic enzymes, omeprazole, ranitidine, antibiotics, digoxin, antihypertensive drugs, metoclopramid, iron, red cell and fresh frozen plasma transfusions. Additionally patient underwent intensive rehabilitation treatment, resulting in enhanced functional outcomes.

After 30 days of hospitalization the patient was discharged from Clinic of Gastroenterology and his condition was described as a satisfactory. Abdominal sonography for follow-up examination showed gradual lowering of the cysts size.

\section{DISCUSSION}

The paper presents the case of severe biliary AP with systemic and local complications (kidney failure, uremic encephalopathy, respiratory failure, diffuse peritonitis, septicemia, pancreatic necrosis, and fluid collections) and management during 41 days of hospitalization. It is worth underlining several contributing factors to the severity of the disease, length and costs of hospitalization, and prediction of survival and recovery.

Severe cases of AP, associated with an organ failure are quite rare, but morality rate in necrotizing type of AP is high and ranges $8-39 \%$, comparing with $2-9 \%$ in all cases [2]. There is no doubt that surgical intervention in biliary AP is beneficial for the patients, and that in severe biliary AP emergency endoscopic retrograde cholangiopancreatography (ERCP) is mandatory. The method and timing of surgery depends on the severity of the disease and should be chosen using scoring systems (e.g. APACHE scale, Ranson criteria). Most authors indicate early sphincterectomy, and delayed (by weeks, months) laparoscopic cholecystectomy for severe AP as a standard $[3,4]$. This surgical delay allows recovery from severe $\mathrm{AP}$ and can also coincide with procedures for internal drainage of pseudocyst or removal of necrotizing tissue. Moreover, in patients with severe pancreatitis, early open cholecystectomy is associated with much higher morbidity and mortality as compared with delayed laparoscopic surgery [5]. Surprisingly, the patient was treated with open cholecystectomy at admission, and ERCP was not performed. Such management could possibly affect further development of AP complications, worsen patient's general condition, prolong hospital length of stay, and, finally, markedly increase costs of hospitalization. Lack of energetic support within early phase of treatment is another striking fact of this case. Acute pancreatitis is a disease related with highly, and in severe form even dramatically developed catabolic processes characterized by energy expenditure, protein breakdown, and substrate utilization. As a result patients loose weight, their condition worsens and the risk of morbidity and mortality increases because of development of multiple organ dysfunction syndrome and sepsis. There is a substantial body of evidence that early enteral nutrition (EN) in severe AP is not only nutritional support, but also a treatment that minimalizes the severity of the inflammatory injury, and prevents bacterial or endotoxin translocation, and septic complications, which may occur when total parenteral nutrition (TPN) is used. Enteral nutrition is well tolerated and does not stimulate increased pancreatic secretion. Moreover, EN has potential benefits in terms of reduced costs - the cost of daily treatment is 20 USD for EN and 200 USD for TPN. Thus, when EN is possible, TPN should be avoided in severe AP [6].

To decrease infectious complications of severe AP and its high mortality, patients should be treated with 
broad-spectrum antibiotics as a prophylaxis. The advantage is limited to patients with severe AP who receive broad-spectrum antibiotics that achieve therapeutic pancreatic tissue levels. It is worth mentioning that fungal infection is also a relatively common complication, so it seems logical that antifungal prophylaxis should be a part of combined management (together with antibiotics). In this case patient received most effective agents: cilastin, metronidazole, doxycycline and fluconazole. In the ED patient was treated with three antioxidants: acetylcysteine, ascorbic acid, and pentoxiphillin, a drug that additionally inhibits secretion of proinflammatory cytokines. It is important to remember that generation of proinflammatory cytokines and free radicals in AP leads to the local and systemic complications such as respiratory failure, renal dysfunction, gastrointestinal ischemia with bacterial translocation, and/or systemic inflammatory response syndrome. Thus, the therapy with antioxidants accompanied with conventional management seems to be reasonable, although the literature shows contradictory findings $[7,8]$.

Finally, we want to comment one of the AP complications - the pancreatic pseudocysts. In this case fluid collections and pseudocysts were not surgically removed and the patient was treated conservatively. It is hard to discuss with such management, since surgical drainage, even if the cyst is large, still remains therapeutic dilemma and there is no consensus regarding methods of intervention if pancreatic pseudocyst is asymptomatic and not related with further complications. Some of the reports recommend surgical drainage for large-sized cysts after six weeks from the onset of AP, while the others indicate that the cysts should be drained only if uncontrolled pain or gastric outlet obstruction develop $[9,10]$.

In summary, we compared described case with Practice Guidelines in Acute Pancreatitis [1], and concluded: (1) severity assessment was not done properly and regularly (no contrast-enhanced CT scan 3 days after the onset of symptoms; in this case: at admission), (2) ERCP with endosocopic biliary sphincterotomy and stone removal, and/or delayed laparoscopic cholecystectomy were not performed, instead patient underwent open surgery, (3) supportive care should include aggressive fluid resuscitation, immediate oxygen supply, and enteral nutritional support as soon as possible - these interventions were not started soon enough, (4) patients with acute pancreatitis caused by gallstones require careful assessment according to current established recommendations.

\section{DISCLOSURE}

Authors report no conflict of interest.

\section{References}

1. Banks PA, Bollen TL, Dervenis C, et al. Classification of acute pancreatitis - 2012: revision of the Atlanta classification and definitions by international consensus. Gut 2013; 62: 102-111.

2. Banks PA, Freeman ML. Practice guidelines in acute pancreatitis. Am J Gastroenterol 2006; 101: 2379-2400.

3. Heinrich S, Schafer M, Rousson V, Clavien PA. Evidence-based treatment of acute pancreatitis: a look at established paradigms. Ann Surg 2006; 243: 154-168.

4. Wilson CT, de Moya MA. Cholecystectomy for acute gallstone pancreatitis: early vs. delayed approach. Scand J Surg 2010; 99: 81-85.

5. Nealon WH, Bawduniak J, Walser EM. Appropriate timing of cholecystectomy in patients who present with moderate to severe gallstone-associated acute pancreatitis with peripancreatic fluid collections. Ann Surg 2004; 239: 741-749.

6. Kanwar S, Windsor A, Reynolds JV. Early nutrition in acute pancreatitis. Nutrition 1999; 15: 951-952.

7. Eggimann P, Jamdar S, Siriwardena AK. Pro/con debate: Antifungal prophylaxis is important to prevent fungal infection in patients with acute necrotizing pancreatitis receiving broad-spectrum antibiotics. Crit Care 2006; 10: 229.

8. Schoenberg MH, Buchler M, Gaspar M, et al. Oxygen free radicals in acute pancreatitis of the rat. Gut 1990; 31: 1138-1143.

9. Vitas GJ, Sarr MG. Selected management of pancreatic pseudocysts: operative versus expectant management. Surgery 1992; 111: 123-130.

10. Aghdassi A, Mayerle J, Kraft M, et al. Pancreatic pseudocysts when and how to treat? HPB (Oxford) 2006; 8: 432-441. 